\title{
EINSTEIN METRICS AND MOSTOW RIGIDITY
}

\author{
Claude LeBrun
}

\begin{abstract}
Using the new diffeomorphism invariants of Seiberg and Witten, a uniqueness theorem is proved for Einstein metrics on compact quotients of irreducible 4-dimensional symmetric spaces of non-compact type. The proof also yields a Riemannian version of the Miyaoka-Yau inequality.
\end{abstract}

A smooth Riemannian manifold $(M, g)$ is said [1] to be Einstein if its Ricci curvature is a constant multiple of $g$. Any irreducible locallysymmetric space is Einstein, and, in light of Mostow rigidity [5], it is natural to ask whether, up to diffeomorphisms and rescalings, the standard metric is the only Einstein metric on any compact quotient of an irreducible symmetric space of non-compact type and dimension $>2$. For example, any Einstein 3-manifold has constant curvature, so the answer is certainly affirmative in dimension 3 . In dimension $\geq 4$, however, solutions to Einstein's equations can be quite non-trivial. Nonetheless, the following 4-dimensional result was recently proved by means of an entropy comparison theorem [2]:

Theorem 1 (Besson-Courtois-Gallot). Let $M^{4}$ be a smooth compact quotient of hyperbolic 4-space $\mathcal{H}^{4}=S O(4,1) / S O(4)$, and let $g_{0}$ be its standard metric of constant sectional curvature. Then every Einstein metric $g$ on $M$ is of the form $g=\lambda \varphi^{*} g_{0}$, where $\varphi: M \rightarrow M$ is a diffeomorphism and $\lambda>0$ is a constant.

In this note, we will prove the analogous result for the remaining 4dimensional cases:

Theorem 2. Let $M^{4}$ be a smooth compact quotient of complex-hyperbolic 2-space $\mathbb{C} \mathcal{H}_{2}=S U(2,1) / U(2)$. Let $g_{0}$ be its standard complex-hyperbolic metric. Then every Einstein metric $g$ on $M$ is of the form $g=\lambda \varphi^{*} g_{0}$, where $\varphi: M \rightarrow M$ is a diffeomorphism and $\lambda>0$ is a constant.

In contrast to Theorem 1, the proof of this result is based on the new 4-manifold invariants [4] recently introduced by Witten [6].

Received November 20, 1994

Supported in part by NSF grant DMS-9003263. 


\section{Seiberg-Witten invariants}

While the results in this section are largely due to Edward Witten [6], the crucial sharp form of the scalar-curvature inequality was pointed out to the author by Peter Kronheimer.

Let $(M, g)$ be a smooth compact Riemannian manifold, and suppose that $M$ admits an almost-complex structure. Then the given component of the almost-complex structures on $M$ contains almost-complex structures $J: T M \rightarrow T M, J^{2}=-1$ which are compatible with $g$ in the sense that $J^{*} g=g$. Fixing such a $J$, the tangent bundle $T M$ of $M$ may be given the structure of a rank-2 complex vector bundle $T^{1,0}$ by defining scalar multiplication by $i$ to be $J$. Setting $\wedge^{0, p}:=\wedge^{p}{\overline{T^{1,0}}}^{*} \cong \wedge^{p} T^{1,0}$, we may then define rank-2 complex vector bundles $V_{ \pm}$on $M$ by

$$
\begin{aligned}
& V_{+}=\wedge^{0,0} \oplus \wedge^{0,2} \\
& V_{-}=\wedge^{0,1},
\end{aligned}
$$

and $g$ induces canonical Hermitian inner products on these bundles.

As described, these bundles depend on the choice of a particular almostcomplex structure $J$, but they have a deeper meaning [3] that depends only on the homotopy class $c$ of $J$; namely, if we restrict to a contractible open set $U \subset M$, the bundles $V_{ \pm}$may be canonically identified with $\mathbb{S}_{ \pm} \otimes L^{1 / 2}$, where $\mathbb{S}_{ \pm}$are the left- and right-handed spinor bundles of $g$, and $L^{1 / 2}$ is a complex line bundle whose square is the 'anti-canonical' line-bundle $L=\left(\overline{\wedge^{0,2}}\right)^{*} \cong \wedge^{0,2}$. For each connection $A$ on $L$ compatible with the $g$ induced inner product, we can thus define a corresponding Dirac operator

$$
D_{A}: C^{\infty}\left(V_{+}\right) \rightarrow C^{\infty}\left(V_{-}\right) .
$$

If $J$ is parallel with respect to $g$, so that $(M, g, J)$ is a Kähler manifold, and if $A$ is the Chern connection on the anti-canonical bundle $L$, then $D_{A}=\sqrt{2}\left(\bar{\partial} \oplus \bar{\partial}^{*}\right)$, where $\bar{\partial}: C^{\infty}\left(\wedge^{0,0}\right) \rightarrow C^{\infty}\left(\wedge^{0,1}\right)$ is the $J$-antilinear part of the exterior differential $d$, acting on complex-valued functions, and where $\bar{\partial}^{*}: C^{\infty}\left(\wedge^{0,2}\right) \rightarrow C^{\infty}\left(\wedge^{0,1}\right)$ is the formal adjoint of the map induced by the exterior differential $d$ acting on 1-forms; more generally, $D_{A}$ will differ from $\sqrt{2}\left(\bar{\partial} \oplus \bar{\partial}^{*}\right)$ by only $0^{\text {th }}$ order terms.

The Seiberg-Witten equations

$$
\begin{aligned}
D_{A} \Phi & =0 \\
F_{A}^{+} & =i \sigma(\Phi) .
\end{aligned}
$$

are equations for an unknown smooth connection $A$ on $L$ and an unknown smooth section $\Phi$ of $V_{+}$. Here the purely imaginary 2 -form $F_{A}^{+}$is the selfdual part of the curvature of $A$, and, in terms of (1), the real-quadratic 
map $\sigma: V_{+} \rightarrow \wedge_{+}^{2}$ is given by

$$
\sigma(f, \phi)=\left(|f|^{2}-|\phi|^{2}\right) \frac{\omega}{4}+\Im m(\bar{f} \phi),
$$

where $\omega(\cdot, \cdot)=g(J \cdot, \cdot)$ is the 'Kähler' form. Notice that $\left|F^{+}\right|=2^{-3 / 2}|\Phi|^{2}$.

For each solution $(A, \Phi)$ of (3) and (4), one can generate a new solution $(A+2 d \log f, f \Phi)$ for any $f: M \rightarrow S^{1} \subset \mathbb{C}$; two solutions which are related in this way are called gauge equivalent, and may be considered to be geometrically identical. A solution is called reducible if $\Phi \equiv 0$; otherwise, it is called irreducible.

A useful generalization of the Seiberg-Witten equations is obtained by replacing (4) with the equation

$$
i F^{+}+\sigma(\Phi)=\varepsilon
$$

for an arbitrary $\varepsilon \in C^{\infty}\left(\wedge^{+}\right)$. We can then consider the map which sends solutions of (3) and (5) to the corresponding $\varepsilon \in C^{\infty}\left(\wedge^{+}\right)$, and define a solution to be transverse if it is a regular point of this map, i.e. if the linearization $C^{\infty}\left(V_{+} \oplus \wedge^{1}\right) \rightarrow C^{\infty}\left(\wedge_{+}^{2}\right)$ of the left-hand-side of (5), constrained by the linearization of (3), is surjective.

Example. Let $(M, g, J)$ be a Kähler surface of constant scalar curvature $s<0$. Let $\Phi=(\sqrt{-s}, 0) \in \wedge^{0,0} \oplus \wedge^{0,2}$, and let $A$ be the Chern connection on the anti-canonical bundle. Since $F_{A}^{+}=-i s \omega / 4,(\Phi, A)$ is an irreducible solution of the Seiberg-Witten equations (3) and (4).

The linearization of (3) at this solution is just

$$
\left(\bar{\partial} \oplus \bar{\partial}^{*}\right)(u+\psi)=-\frac{\sqrt{-s}}{2} \alpha,
$$

where $(u, \psi) \in C^{\infty}\left(V_{+}\right)$is the linearization of $\Phi=(f, \phi)$ and $\alpha \in \wedge^{0,1}$ is the $(0,1)$-part of the purely imaginary 1 -form which is the linearization of $A$. Linearizing (5) at our solution yields the operator

$$
(u, \psi, \alpha) \mapsto i d^{+}(\alpha-\bar{\alpha})+\frac{\sqrt{-s}}{2}(\Re e u) \omega+\sqrt{-s} \Im m \psi .
$$

Since the right-hand-side is a real self-dual form, it is completely characterized by its component in the $\omega$ direction and its (0,2)-part. The $\omega$-component of this operator is just

$$
(u, \psi, \alpha) \mapsto \Re e\left[-\bar{\partial}^{*} \alpha+\frac{\sqrt{-s}}{2} u\right],
$$


while the $(0,2)$-component is

$$
(u, \psi, \alpha) \mapsto i \bar{\partial} \alpha-i \frac{\sqrt{-s}}{2} \psi .
$$

Substituting (6) into these expressions, we obtain the operator

$$
\begin{aligned}
C^{\infty}\left(\mathbb{C} \oplus \wedge^{0,2}\right) & \longrightarrow C^{\infty}\left(\mathbb{R} \oplus \wedge^{0,2}\right) \\
(u, \psi) & \mapsto\left(\frac{1}{\sqrt{-s}} \Re e\left[\Delta-\frac{s}{2}\right] u,-\frac{i}{\sqrt{-s}}\left[\Delta-\frac{s}{2}\right] \psi\right),
\end{aligned}
$$

which is surjective because $s / 2<0$ is not in the spectrum of the Laplacian. The constructed solution is therefore transverse.

Relative to $c=[J]$, a metric $g$ will be called excellent if it admits only irreducible transverse solutions of (3) and (4). Relative to any excellent metric, the set of solutions of (3) and (4), modulo gauge equivalence, is finite $[4,6]$. Notice that a metric $g$ is automatically excellent if the corresponding equations (3) and (4) admit no solutions at all.

Definition 1. Let $(M, c)$ be a compact 4-manifold equipped with a a homotopy class $c=[J]$ of almost-complex structures. Assume either

$$
\begin{gathered}
b_{+}(M)>1 \\
\text { or } \\
b_{+}=1 \quad \text { and } \quad(2 \chi+3 \tau)(M)>0 .
\end{gathered}
$$

If $g$ is an excellent metric on $M$, define the (mod 2) Seiberg-Witten invariant $n_{c}(M) \in \mathbb{Z}_{2}$ to be

$$
n_{c}(M)=\#\{\text { gauge classes of solutions of (3) and (4) }\} \bmod 2
$$

calculated with respect to $g$.

It turns out [4] that $n_{c}(M)$ is actually metric-independent; when $b_{+}=1$, this fact depends on the assumption that $c_{1}(L)^{2}=2 \chi+3 \tau>0$, which guarantees that (3) and (4) cannot admit reducible solutions for any metric.

Theorem 3. Let $(M, J)$ be a compact complex surface, where the underlying oriented 4-manifold $M$ is as in Definition 1. Suppose that $(M, J)$ admits a Kähler metric $g$ of constant scalar curvature $s<0$, and let $c=[J]$. Then $n_{c}(M)=1 \in \mathbb{Z}_{2}$. 
Proof. With respect to $g$ we shall show that, up to gauge equivalence, there is exactly one solution of the Seiberg-Witten equations, namely the one described in the above example. Indeed, the Weitzenböck formula for the twisted Dirac operator and equation (4) tell us that

$$
0=D_{A}^{*} D_{A} \Phi=\nabla^{*} \nabla \Phi+\frac{s}{4} \Phi+\frac{1}{4}|\Phi|^{2} \Phi,
$$

which implies [4] the $C^{0}$ estimate $|\Phi|^{2} \leq-s$, with equality only at points where $\nabla \Phi=0$. Since

$$
\left|F_{A}^{+}\right|^{2}=\frac{1}{8}|\Phi|^{4} \leq \frac{s^{2}}{8}
$$

it follows that

$$
\int_{M}\left|F_{A}^{+}\right|^{2} d \mu \leq \int_{M}\left(\frac{s}{4}|\omega|\right)^{2} d \mu=\int_{M}\left|\rho^{+}\right|^{2} d \mu
$$

where the Ricci form $\rho$ is in the same cohomology class as the closed form $F_{A}$, namely $2 \pi c_{1}(L)=2 \pi c_{1}(M, J)$. But since $s$ is constant, $\rho$ is harmonic, and we must therefore have that

$$
\begin{aligned}
\int_{M}\left|\rho^{+}\right|^{2} d \mu & =2 \pi^{2} c_{1}(L)^{2}+\frac{1}{2} \int_{M}|\rho|^{2} d \mu \\
& \leq 2 \pi^{2} c_{1}(L)^{2}+\frac{1}{2} \int_{M}\left|F_{A}\right|^{2} d \mu \\
& =\int_{M}\left|F_{A}^{+}\right|^{2} d \mu
\end{aligned}
$$

because a harmonic form minimizes the $L^{2}$ norm among closed forms in its deRham class. Hence $F_{A}=\rho$, and $A$ differs from the Chern connection on $L$ by twisting with a flat connection. But also $|\Phi|^{2} \equiv-s$, which forces $\nabla \Phi \equiv 0$. Since $c_{1}(L) \neq 0$, the induced connection on $\wedge^{0,2} \subset V_{+}$has nontrivial curvature, and $\Phi$ must therefore be a section of $\wedge^{0,0}$. Since $\Phi$ is parallel, the induced connection on $\wedge^{0,0}$ must not only be flat, but also have trivial holonomy. Thus $A$ must exactly be the Chern connection on $L$, and our solution coincides, up to gauge transformation, with that of the example. In particular, every solution with respect to $g$ is irreducible and transverse, so $g$ is excellent. But since there is only one gauge class of solutions with respect to $g$, we conclude that $n_{c}(M)=1 \bmod 2$.

The following refinement of an observation of Witten $[6, \S 3]$ is the real key to the proof of Theorem 2 .

Theorem 4. Let $M$ be a smooth compact oriented 4-manifold with

$$
2 \chi(M)+3 \tau(M)>0 .
$$


Suppose that there is a an orientation-compatible class $c=[J]$ of almostcomplex structures for which the Seiberg-Witten invariant $n_{c}(M) \in \mathbb{Z}_{2}$ is non-zero. Let $g$ be a metric of constant scalar curvature $s$ and volume $V$ on $M$. Then

$$
s \sqrt{V} \leq-2^{5 / 2} \pi \sqrt{2 \chi+3 \tau}
$$

with equality iff $g$ is Kähler-Einstein with respect to some integrable complex structure $J$ in the homotopy class $c$.

Proof. For any given metric $g$ on $M$, there must exist a solution of (3) and (4), since otherwise we would have $n_{c}(M)=0$. But since $\left|F_{A}^{+}\right|^{2}=$ $|\Phi|^{4} / 8 \leq s^{2} / 8$, with equality iff $\nabla \Phi=0$, it follows that

$$
2 \chi+3 \tau=c_{1}(L)^{2}=\frac{1}{4 \pi^{2}} \int_{M}\left(\left|F_{A}^{+}\right|^{2}-\left|F_{A}^{-}\right|^{2}\right) d \mu \leq \frac{1}{32 \pi^{2}} \int_{M} s^{2} d \mu,
$$

with equality only if

$$
\nabla F_{A}^{+} \equiv 0 \quad \text { and } \quad F_{A}^{-}=0 .
$$

If equality holds, the parallel self-dual form

$$
\sqrt{2} F_{A} /\left|F_{A}\right|
$$

corresponds via $g$ to a parallel almost-complex structure $J$, and the manifold is thus Kähler, with Kähler class $8 \pi / s$ times $c_{1}(M, J)=c_{1}(L)$. But since $s$ is constant, the Ricci form is harmonic, and the manifold is KählerEinstein.

On the other hand, any Kähler-Einstein metric will saturate the bound in question, since the first Chern class of a Kähler-Einstein surface is $[s \omega / 8 \pi]$, and the metric volume form is $d \mu=\omega^{2} / 2$.

\section{The Miyaoka-Yau inequality}

For any compact oriented Riemannian 4-manifold $(M, g)$, the Euler characteristic and signature can be expressed as

$$
\begin{gathered}
\chi(M)=\frac{1}{8 \pi^{2}} \int_{M}\left(\left|W_{+}\right|^{2}+\left|W_{-}\right|^{2}+\frac{s^{2}}{24}-\frac{\mid \text { ric }\left._{0}\right|^{2}}{2}\right) d \mu \\
\tau(M)=\frac{1}{12 \pi^{2}} \int_{M}\left(\left|W_{+}\right|^{2}-\left|W_{-}\right|^{2}\right) d \mu
\end{gathered}
$$

where $s, \operatorname{ric}_{0}, W_{+}$and $W_{-}$are respectively the scalar, trace-free Ricci, selfdual Weyl, and anti-self-dual Weyl parts of the curvature tensor; pointwise 
norms are calculated with respect to $g$, and $d \mu$ is the metric volume form. If $g$ is Einstein, ric $_{0}=0$, and $M$ therefore satisfies

$$
(2 \chi \pm 3 \tau)(M)=\frac{1}{4 \pi^{2}} \int_{M}\left(2\left|W_{ \pm}\right|^{2}+\frac{s^{2}}{24}\right) d \mu,
$$

so the Hitchin-Thorpe inequality $2 \chi+3 \tau \geq 0$ holds, with strict inequality unless $M$ is finitely covered by a 4 -torus or $K 3$ surface.

Now assume that $M$ admits a homotopy class of almost-complex structures for which the Seiberg-Witten invariant is non-zero. If $g$ is an Einstein metric on $M$, Theorem 4 then tells us that

$$
\begin{aligned}
2 \chi+3 \tau & \leq \frac{1}{32 \pi^{2}} \int_{M} s^{2} d \mu \\
& \leq 3\left[\frac{1}{4 \pi^{2}} \int_{M}\left(\left|2 W_{-}\right|^{2}+\frac{s^{2}}{24}\right) d \mu\right] \\
& =3(2 \chi-3 \tau)
\end{aligned}
$$

with equality iff the metric is Kähler and $W_{-}=0$. But the curvature operator of any Kähler manifold is an element of $\wedge^{1,1} \otimes \wedge^{1,1}$, and in real dimension 4 one also has $\wedge^{1,1}=\wedge^{-} \oplus \mathbb{C} \omega$, where $\omega$ is the Kähler form; when

$$
W_{-}: \wedge_{-} \rightarrow \wedge_{-} \quad \text { and } \quad \operatorname{ric}_{0}: \wedge_{-} \rightarrow \wedge_{+}
$$

both vanish, the curvature operator must therefore be of the form

$$
\mathcal{R}=\frac{s}{8} \omega \otimes \omega+\frac{s}{12} 1_{\wedge_{-}}
$$

and so satisfy

$$
\nabla \mathcal{R}=0
$$

which is to say that $(M, g)$ must be locally symmetric. Unless $g$ is flat, the non-triviality of the Seiberg-Witten invariant now forces $s$ to be negative, and the point-wise form of the curvature tensor then implies that the exponential map induces an isometry between the universal cover of $(M, g)$ and a complex-hyperbolic space which has been rescaled so as to have the same scalar curvature. This proves the following generalization of the Miyaoka-Yau inequality [7]:

Theorem 5. Let $(M, g)$ be a compact Einstein 4-manifold, and suppose that $M$ admits an almost-complex structure $J$ for which the Seiberg-Witten invariant is non-zero. Also assume that $M$ is not finitely covered by the 
4-torus $T^{4}$. Then, with respect to the orientation of $M$ determined by $J$, the Euler characteristic and signature of $M$ satisfy

$$
\chi \geq 3 \tau,
$$

with equality iff the universal cover of $(M, g)$ is complex-hyperbolic 2-space $\mathbb{C H}_{2}:=S U(2,1) / U(2)$ with a constant multiple of its standard metric.

On the other hand, Theorem 3 tells us the Seiberg-Witten invariant of any complex hyperbolic 4-manifold $M=\mathbb{C H}_{2} / \Gamma$ is actually non-zero. Theorem 5 and Mostow rigidity thus imply Theorem 2.

\section{Acknowledgement}

The author would like to express his gratitude to Peter Kronheimer for the e-mail exchanges which made this paper possible.

\section{References}

1. A. Besse, Einstein Manifolds, Springer-Verlag, 1987.

2. G. Besson, G. Courtois, and S. Gallot, Entropies et Rigidités des Espaces Localement Symétriques de Courbure Strictement Négative, GAFA, to appear.

3. N.J. Hitchin, Harmonic Spinors, Adv. Math. 14 (1974) 1-55.

4. P. Kronheimer and T. Mrowka, The Genus of Embedded Surfaces in the Complex Projective Plane, Math. Res. Letters 1 (1994) 797-808.

5. G.D. Mostow, Quasi-Conformal Mappings in n-Space and the Rigidity of Hyperbolic Space Forms, Publ. IHES 34 (1968) 53-104.

6. E. Witten, Monopoles and Four-Manifolds, Math. Res. Letters 1 (1994) 769-796.

7. S.-T. Yau, Calabi's Conjecture and Some New Results in Algebraic Geometry, Proc. Nat. Acad. USA74 (1977) 1789-1799.

Department of Mathematics, State University of New York, Stony Brook, NY 11794-3651

E-mail address: claude@math.sunysb.edu 